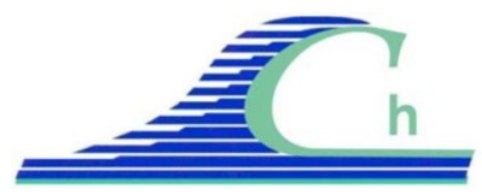

XII ${ }^{\text {èmes }}$ Journées Nationales Génie Côtier - Génie Civil

Cherbourg, 12-14 juin 2012

DOI:10.5150/jngcgc.2012.113-L C Editions Paralia CFL

disponible en ligne - http://www.paralia.fr - available online

\title{
Gestion durable des sédiments : co-valorisation sédiments-fibres
}

\author{
Daniel LEVACHER ${ }^{1}$, Bruna BATAGIN ${ }^{1,2}$, Yingjie LIANG $^{1}$, Nabil CHERIFI ${ }^{1}$ \\ 1. Université de Caen, Laboratoire M2C, UMR 6143 CNRS, 24 rue des Tilleuls, \\ 14000 Caen, France. \\ daniel.levacher@unicaen.fr,yingjie.liang@unicaen.fr
}

2. Environmental Engineering Faculty, Engineering University of Piracicaba, Brazil DF. bbatagin@gmail.com

\section{Résumé :}

Dans un contexte de gestion durable de déchets solides, des solutions de valorisation de sols fins à problèmes (sédiments marins et fillers de carrière) sont recherchées. La filière de valorisation matière retenue est basée sur d'une part, le réemploi de fibres déchets si possible, et d'autre part, la stabilisation à base de liants hydrauliques faiblement dosés. Cette co-valorisation constitue une nouvelle approche innovante dans les traitements des sédiments marins ou fines de carrière avec des ajouts de liants hydrauliques. Une étude préliminaire a porté dans le cas de fibres naturelles sur le caractère absorbant et la mesure du taux d'absorption. Puis une étude sur des mélanges a été entreprise dont l'objectif était la valorisation de sédiments marins de La Baule - Le Pouliguen et de fillers issus de carrières proches de Bayeux. L'application visée concerne l'élaboration d'un matériau pour le domaine routier. Sur ces mélanges, l'analyse a porté sur les performances mécaniques des mélanges fibrés (fibres de lin, fibres géosynthétiques rigides - Kevlar et lisses à base de polypropylène). Les propriétés mécaniques des mélanges sont fonction du choix de la nature, de la quantité de fibres ajoutée et de leur longueur mais aussi de leurs propriétés intrinsèques. Les essais considérés sont la compression simple, la traction indirecte et de mouillage-séchage. Des tendances sur les paramètres optimaux ont été mises en évidence.

\section{Mots-clés :}

Sédiments - Filler - Fibres naturelles - Fibres géosynthétiques - Valorisation Matériau - Stabilisation/solidification - Compression - Traction - Gestion durable

\section{Introduction}

Une alternative au clapage des sédiments issus de dragage des zones portuaires dont les volumes extraits sont importants (AIPCN, 2006) et au stockage des fillers issus de l'exploitation des carrières, nécessite la recherche de voies nouvelles de valorisation pour ces sols fins. Ces filières de valorisation doivent intégrer la notion de coût économique pour traiter et éliminer ces matériaux stockés, considérer les enjeux 
environnementaux et sociétaux et générer des revenus quant à leur réutilisation en tant que matériaux alternatifs ou recyclés.

La solidification et/ou stabilisation à l'aide de ciment ou de chaux est une méthode largement éprouvée pour améliorer les performances mécaniques des sols fins pour une application en construction routière (SETRA \& LCPC, 2000). Ces additions parfois confèrent aux sols traités un comportement fragile. On peut aussi améliorer les propriétés mécaniques des sols fins en procédant à l'intégration de fibres de renforcement. Cette technique est efficace et fiable (LÊ, 2009; TANG et al., 2007 ; KOTAKE et al., 2012). Il a donc été tenté d'associer ces deux méthodes d'amélioration pour une valorisation matière de sédiments et de fillers avec ajout de liants faiblement dosés et de fibres naturelles et géosynthétiques. Les premières applications de renforcement de sols fins par des fibres naturelles datent des années 60, avec des nappes de coton écru tissé incluses dans des sous couches asphaltées aux USA. SERFASS et SAMANOS (1996) ont étudié les effets dus aux fibres ajoutées dans ce type de matrice : fibres d'amiante, de laine de verre et de cellulose. L'utilisation de fibres naturelles réduit le poids volumique et la demande d'énergie au niveau de leur production. Elles sont aussi moins chères que les fibres géosynthétiques et de verre mais elles n'atteignent pas les mêmes performances mécaniques de ces dernières (GEETHAMMA et al., 1998). Par ailleurs les fibres naturelles sont hydrophiles et présentent une faible capacité de résistance à la température et des qualités variables au niveau de la production. Les fibres naturelles absorbent de l'eau et de l'air, ce qui peut provoquer des phénomènes de gonflement-retrait et créer des pores au sein des matrices des mélanges (GHAVAMI et al., 1999). Ceci peut diminuer les résistances des mélanges sols fins - liants - fibres en raison du volume de pores d'autant plus important que la structure de la fibre est multifilaire. Des mesures relatives à l'absorption d'eau ont été entreprises sur différentes fibres susceptibles d'être valorisées comme renforcement dans des mélanges de sédiments et de fillers. Il s'agit de fibres naturelles : de palmiers à huile, de lin et de chanvre. Elles sont rapportées ici.

Puis une étude préliminaire de co-valorisation a été menée sur des sédiments marins issus de La Baule - Le Pouliguen et sur un sol fin équivalent : un filler de carrière de Vaubadon (Bayeux, Calvados). Pour cette étude ont été ajoutées des fibres végétales et artificielles dans une stabilisation à base de liants hydrauliques (CHERIFI, 2011).

\section{Le concept Sédifibres ${ }^{\circledR}$}

Le concept Sédifibres ${ }^{\circledR}$ consiste à associer des sédiments ou des sols fins équivalents par mélanges avec des liants hydrauliques, pouzzolaniques, chimiques, éco compatibles et des géopolymères en vue de les stabiliser ou solidifier avec des fibres naturelles et/ou artificielles. Le produit qui résulte de ces mélanges est un matériau valorisable en génie civil, en travaux publics et maritimes et en aménagements. Les liants utilisés correspondent à tous ceux disponibles sur le marché. 


\section{XII ${ }^{\text {èmes }}$ Journées Nationales Génie Côtier - Génie Civil \\ Cherbourg, 12-14 juin 2012}

Les fibres rigides ou souples, de longueur variable, sont d'origine naturelle ou artificielle. Elles peuvent être brutes, élaborées ou déchets. Elles concernent tous les additifs fibreux. Les sédiments sont marins, fluviaux comme issus de voies d'eau. Les sols fins équivalents sont des fillers ou sables de carrière non commercialisables considérés comme déchets. Les mélanges Sédifibres ${ }^{\circledR}$ englobent toutes les formes de matériau : volume à géométrie variable (remplissage), surface (couche), volumes finis (produits élaborés), quels qu'en soient le procédé de mise en œuvre et l'application (voir figure 1).

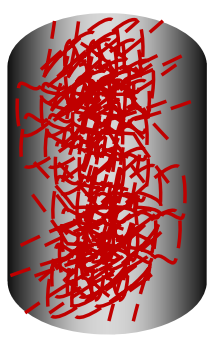

Formes préfabriquées (briques, ...)

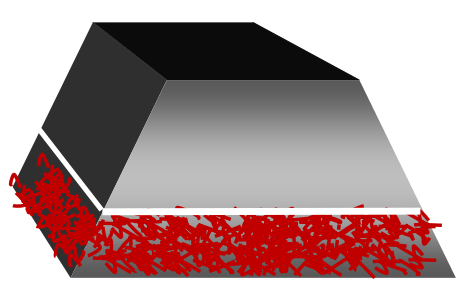

Couches de remblai (routes) Recifs artificiels

Figure 1.Formes issues de mélanges stabilisés et/ou solidifiés de type Sédifibres ${ }^{\circledR}$.

\section{Fibres naturelles et géosynthétiques}

Dans le monde, le nombre de fibres naturelles potentielles pour un renforcement de sols fins stabilisés est important et leur nature dépend beaucoup de l'activité agricole et forestière locale (HEJAZI et al., 2012). Des fibres naturelles ont déjà utilisées par divers auteurs dans des mélanges à matrice cimentaire : du chanvre, (figure 2, WANG, 2008), de la fibre de riz (BASHA et al., 2005), du lin (LEVACHER et al., 2010 ; LEVACHER et al., 2012; LIANG, 2012). Des fibres de fruits peuvent être aussi considérées (figure 2, WANG, 2008).

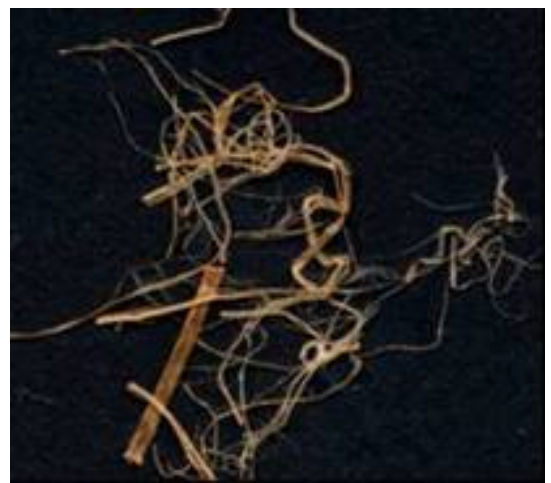

Fibres de bananes

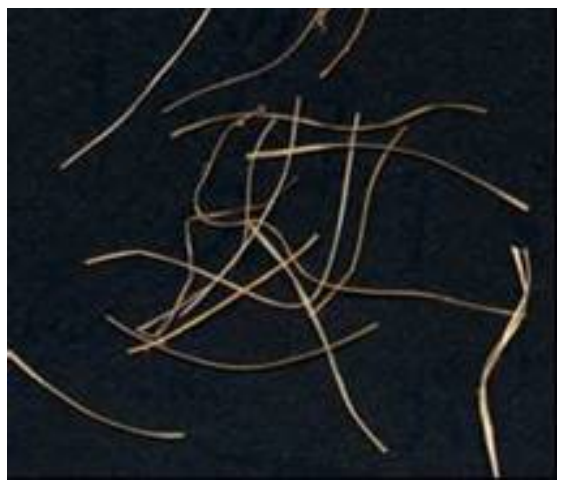

Fibres de chanvre

Figure 2: Fibres végétales souples. 


\subsection{Fibres de lin}

Le lin cultivé (Linum usitatissimum) est une plante annuelle de la famille des Linacées. Le lin possède de longues fibres à parois cellulosique, elle se décompose 3 couches. Les caractéristiques morphologiques sont rappelées au tableau 1.

Tableau 1. Quelques proprietés et composants d'une fibre de lin.

\begin{tabular}{|l|l|l|l|}
\hline \multicolumn{5}{|c|}{ Composition chimique } \\
\hline Cellulose (\%) & $64-74$ & Lignine & $2-3$ \\
\hline Hemicellulose (\%) & $11-17$ & Wax & 1,5 \\
\hline Pectine (\%) & 1,8 & Water & $8-10$ \\
\hline \multicolumn{4}{|c|}{ Caractéristiques physiques et mécaniques } \\
\hline Diamètre moyen d'une fibre $(\mu \mathrm{m})$ & $15-30$ & Module d'élasticité (GPa) & $30-90$ \\
\hline Déformation à la rupture (\%) & $1,5-3,5$ & Résistance à la traction $(\mathrm{MPa})$ & $400-1600$ \\
\hline
\end{tabular}

\subsection{Fibres de palmier à huile}

Le palmier à huile (Elaeis guineensis) est cultivé dans plus de 40 pays sur plus de 11 millions d'hectares. Ses composants chimiques sont donnés au tableau 2.

Tableau 2. Composants d'une fibre de palmier à huile.

\begin{tabular}{|l|l|l|l|}
\hline Cellulose (\%) & $42.7-65$ & Lignine (\%) & $13.2-25.3$ \\
\hline Hemicellulose (\%) & $17.1-33.5$ & Holocellulose (\%) & $68.3-86.3$ \\
\cline { 1 - 2 } Cendres (\%) & $1.3-6.0$ & \multicolumn{2}{|l}{} \\
\cline { 1 - 2 } & \multicolumn{2}{|l}{}
\end{tabular}

\subsection{Fibres de chanvre}

Le chanvre (Cannabis sativa) est composé de brin de fibres élémentaires dont la fibre moyenne est faite de lignine avec des intra-fibres d'hémicellulose. C'est une fibre multicellulaire. La composition chimique (voir tableau 3) est très hétérogène.

Tableau 3. Composants d'une fibre de chanvre.

\begin{tabular}{|l|l|l|l|}
\hline Cellulose (\%) & $67-78$ & Lignine (\%) & $3.7-8$ \\
\hline Hemicellulose (\%) & $5.5-16.1$ & Pectine (\%) & $0.9-4.3$ \\
\hline
\end{tabular}

\subsection{Capacité d'absorption des fibres}

L'absorption d'eau des fibres naturelles est estimée à partir de la mesure de la variation de masse des fibres conformément à la durée d'exposition. Les fibres ont donc été immergées dans un bain d'eau à température ambiante pendant 8 jours. Une mesure a été faite tous les 24 heures après avoir retiré les fibres de l'eau et les avoir égouttées sur du papier. La pesée se fait aussitôt dès que la surface extérieure des fibres paraît sèche (figure 2). 

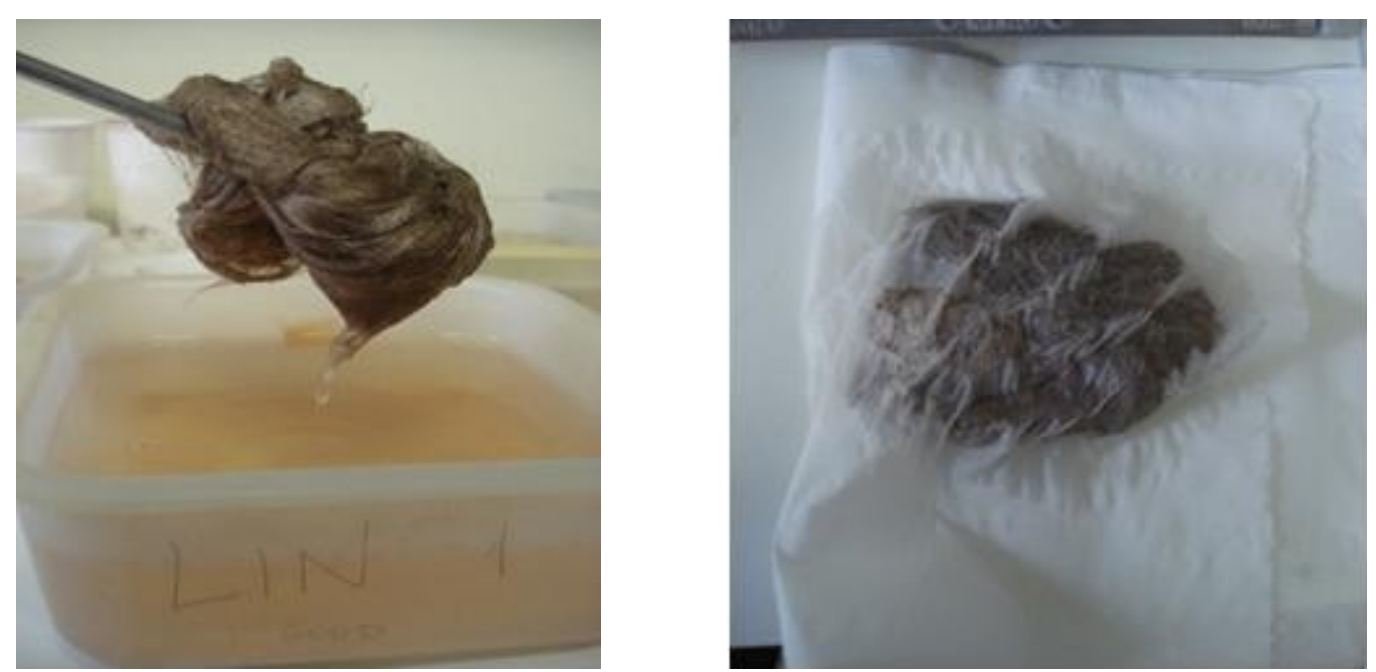

Figure 2. Procédure de l'essai d'absorption, cas du lin : extraction et égouttage

L'évaluation de la teneur en eau $\mathrm{W}$ se fait à partir de la formule classique 1 , où $\mathrm{Ph}$ est le poids humide, $\mathrm{Pd}$ est le poids sec des fibres. Cette valeur peut être retenue comme coefficient d'absorption.

$\mathrm{W}(\%)=[(\mathrm{Ph}-\mathrm{Pd}) / \mathrm{Pd}] \times 100$

On peut observer la variation de l'absorption d'eau dans les fibres étudiées sur la figure 3. Les références correspondent au lin fibre textile (flax 'good'), au lin fibre déchet (flax 'not good'), au chanvre (hemp) et aux fibres de palme (oil palm).

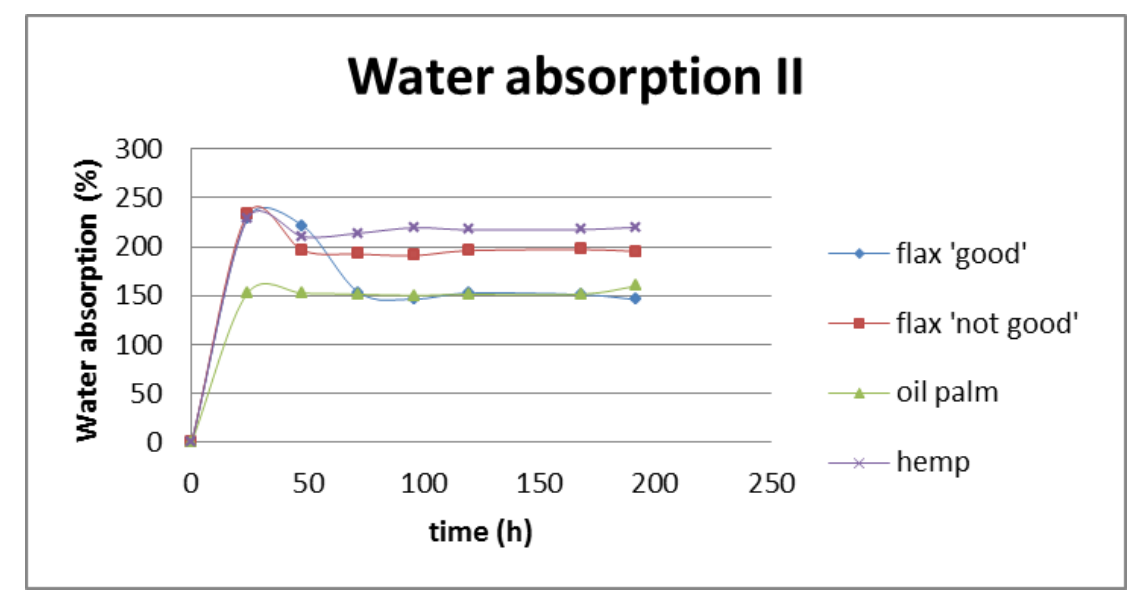

Figure 3. Coefficient d'absorption en fonction du temps

\subsection{Fibres en polypropylène}

La fibre de polypropylène de type PP est une fibre souple, lisse et noire. Elle est utilisée dans la composition de tissus géosynthétiques fibrés. Elle possède une bonne tenue aux produits chimiques en milieu acide ou basique, mais sa tenue à la température est limitée à $80^{\circ} \mathrm{C}$. Le tableau 4 en donne quelques propriétés. Elle sera référencée comme "fibre noire" dans les résultats d'essais. 
Tableau 4. Propriétés et caractéristiques de la fibre polypropylène PP.

\begin{tabular}{|l|l|}
\hline Référence & $P P$ \\
\hline Masse volumique (g/ $\left.\mathrm{cm}^{3}\right)$ & 0,91 \\
\hline Absorption d'humidité (\%) & 0,05 \\
\hline Allongement (\%) & 35 \\
\hline
\end{tabular}

3.6 Fibres à base de kevlar

La fibre en kevlar est une fibre relativement rigide, lisse, torsadée et blanche. Elle est utilisée dans la composition de cordages marins. Le matériau kevlar est une fibre synthétique qui possède de très bonnes propriétés mécaniques, en traction, avec une résistance à la rupture de $3100 \mathrm{MPa}$ et un module d'élasticité compris entre 70 et 125 $\mathrm{GPa}$. Il possède une faible densité de 1,45 . Il absorbe les vibrations et résiste très bien aux chocs. Elle sera référencée comme "fibre blanche" dans les résultats d'essais.

\section{Matériaux fins à stabiliser}

\subsection{Sédiments et fillers}

Les matériaux à valoriser par stabilisation sont des fines de carrière (Bayeux, carrière de Vaubadon, Calvados) et des sédiments de dragage provenant du port de la Baule - Le Pouliguen, situé en bordure côtière Nord de l'estuaire de la Loire en Loire-Atlantique. Les échantillons de sédiments ont été prélevés à partir de deux bassins du port, notés bassin 2 et bassin 3 .

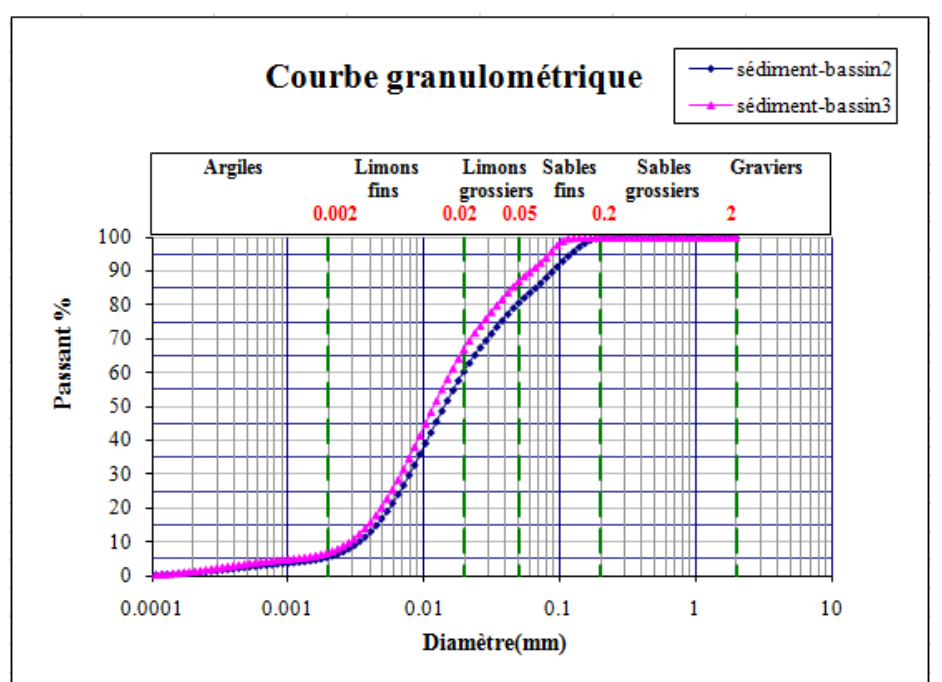

Figure 4. Granulométries des sédiments de La Baule - Le Pouliguen (bassins 2 et 3). 


\section{XII ${ }^{\text {èmes }}$ Journées Nationales Génie Côtier - Génie Civil \\ Cherbourg, 12-14 juin 2012}

Les méthodes de détermination des caractéristiques physiques sont classiques et respectent les normes en vigueur. La finesse des matériaux a permis de réaliser des granulométries laser pour l'ensemble des sédiments et fillers. L'appareil utilisé, un Coulter LS230, permet d'explorer une large gamme de particules allant de 0.04 à $2000 \mu \mathrm{m}$. Les résultats de l'analyse granulométrique effectuée sur les fillers ont montré que $80 \%$ des éléments sont inférieurs à $20 \mu \mathrm{m}$. Pour les sédiments de La Baule - Le Pouliguen (figure 4), on note que les distributions sont très proches quel que soit le bassin et que $70 \%$ des éléments sont inférieurs à $20 \mu \mathrm{m}$.

Quelques mesures relatives aux propriétés physiques et chimiques des sédiments et fillers ont été déterminées pour une valorisation matière. La teneur en eau est obtenue par séchage en étuve à $60{ }^{\circ} \mathrm{C}$ selon la norme (NF P 94-050). Les limites d'Atterberg sont des teneurs en eau qui délimitent des comportements critiques des matériaux : limites de liquidité $\mathrm{W}_{\mathrm{L}}$ et de plasticité $\mathrm{W}_{\mathrm{P}}$ (méthode de la coupelle et du rouleau), selon la norme NF P 94-051. Les teneurs en matières organiques ont été déterminées à partir de la mesure du pourcentage pondéral de matière organique $\mathrm{MO}$ après calcination des matériaux à $550^{\circ} \mathrm{C}$ sur une durée de $4 \mathrm{~h}$ selon la norme XP P 94-047. L'utilisation d'un calcimètre Bernard permet l'obtention des teneurs en carbonates conformément à la norme NF P 94-048. L'ensemble des résultats pour les deux sols fins sont donnés dans le tableau 5.

Tableau 5. Propriétés des matériaux à stabiliser.

\begin{tabular}{|c|c|c|c|c|c|c|c|}
\hline \multicolumn{2}{|c|}{ Matériaux fins } & $\boldsymbol{w}(\%)$ & $\boldsymbol{W}_{\mathbf{L}}(\%)$ & $\boldsymbol{W}_{\boldsymbol{P}}(\%)$ & $\boldsymbol{I}_{\boldsymbol{P}}(\%)$ & $\mathbf{M O}(\%)$ & $\mathrm{CaCO}_{3}(\%)$ \\
\hline \multicolumn{2}{|c|}{ Fillers de carrière } & 29,30 & 34.15 & 28.19 & 5.96 & 0.3 & 3.66 \\
\hline \multirow{2}{*}{ Sédiments de la Baule } & Bassin 2 & 163.91 & 72.27 & 42 & 30.27 & 11.45 & 14.83 \\
\cline { 2 - 8 } & Bassin 3 & 192.36 & 89.25 & 43.01 & 46.23 & 11.59 & 14.11 \\
\hline
\end{tabular}

\subsection{Liant}

Le liant hydraulique est un ciment ordinaire Portland, de type : CEM III/B 32,5 N LH CE PM-ES-CP1 N (tableau 4). Le dosage retenu est de 7\%, dosage exprimé en matières sèches de sédiments ou de fillers.

\section{Méthodologie}

\subsection{Confection des éprouvettes}

Les éprouvettes ont été moulées dans des étuis cylindriques en PVC ayant pour diamètre intérieur $\mathrm{D}$ de $34 \mathrm{~mm}$ et une hauteur de $68 \mathrm{~mm}$. Le compactage est réalisé manuellement par piquetage. Un protocole a été établi pour permettre d'assurer la répétabilité des opérations suivantes: séchage, malaxage, confection et conservation des 
échantillons pour les deux matériaux fins à stabiliser. Pour les fillers, la teneur étant inférieure à celle des mélanges, il n'y a pas eu de phase de séchage. La teneur en eau des mélanges réalisés était de 30\%.

Pour les autres ajouts (fibres) les dosages sont tous exprimés en fonction de la matière sèche. Dans cette étude, la teneur de ciment ajoutée pour les deux matériaux fins a été fixée à $7 \%$. Les fibres sont ajoutées aléatoirement dans le mélange pour chaque série d'éprouvettes dont la formulation dépend du dosage en fibres et de la longueur de fibres. L'objectif de ces essais est d'étudier l'influence de la nature, de la longueur et du dosage des fibres sur la résistance mécanique à partir des éprouvettes confectionnées.

Les paramètres de l'étude sont :

- trois types de fibres : lin, polypropylène et kevlar ;

- trois dosages : $0.2 \%, 0.4 \%, 0.8 \%$;

- des longueurs exprimées en fonction du diamètre $D: D=34 \mathrm{~mm}, D / 2=17 \mathrm{~mm}$, $\mathrm{D} / 4=8.5 \mathrm{~mm}, \mathrm{D} / 8=4.25 \mathrm{~mm}$ ).

La réalisation des différentes combinaisons possibles a donné lieu à la préparation de 72 éprouvettes constituant ainsi une série complète.

\subsection{Protocole des essais de résistance}

Les indicateurs mécaniques retenus dans le cadre de cette étude ont pour but d'évaluer l'intégrité physique et mécanique des mélanges. Pour ce faire, les principaux essais retenus sont la résistance à la compression simple et à la traction. L'estimation de la résistance à la traction est estimée à partir du résultat de l'essai en compression diamétrale ou essai brésilien, selon la norme NF P 982323.

\section{Analyse des résultats}

\subsection{Comportement en résistance à la compression simple}

L'indicateur mécanique de référence est la résistance en compression simple ou non confinée notée Rc. La résistance à la compression simple est aussi le premier essai de contrôle dans l'étude d'une valorisation potentielle en technique routière d'un sédiment traité. Les courbes d'évolution de Rc ont été étudiées. Les discussions portent sur l'influence des différents paramètres d'étude retenus dans les formulations mises en œuvre dans cette étude.

\subsubsection{Evolution Rc en fonction de l'âge}

Pour les fillers, la figure 5a qui est une figure type de l'évolution de Rc avec le temps, le seuil de $1 \mathrm{MPa}$ est largement dépassé, la valeur optimale est généralement obtenue pour les longueurs $\mathrm{D}$ et $\mathrm{D} / 2$ quel que soit le temps. Une amélioration notable des performances mécaniques (Rc) des fines de carrières traitées avec les fibres est observée de 1 'ordre de $10 \%$ jusqu'à $80 \%$ pour certaines formulations. On remarque aussi que Rc 


\section{XII ${ }^{\text {èmes }}$ Journées Nationales Génie Côtier - Génie Civil \\ Cherbourg, 12-14 juin 2012}

atteint généralement son pic à 14 jours avant de diminuer vers 28 jours. L'apport des fibres joue un rôle important dans le phénomène de durcissement et de renforcement.

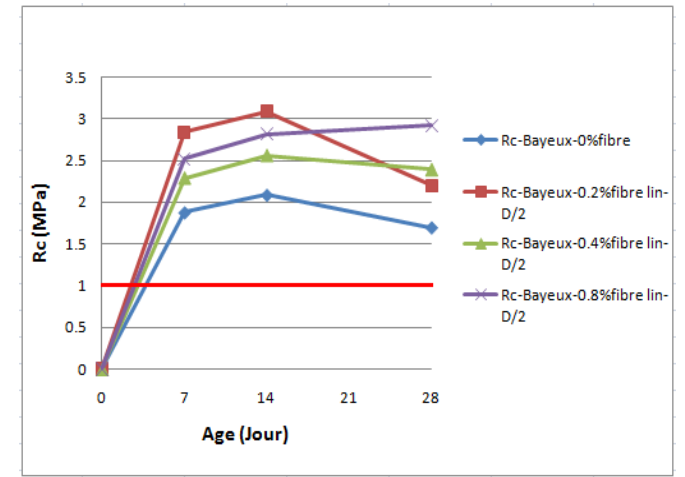

(a) Fillers de carrière

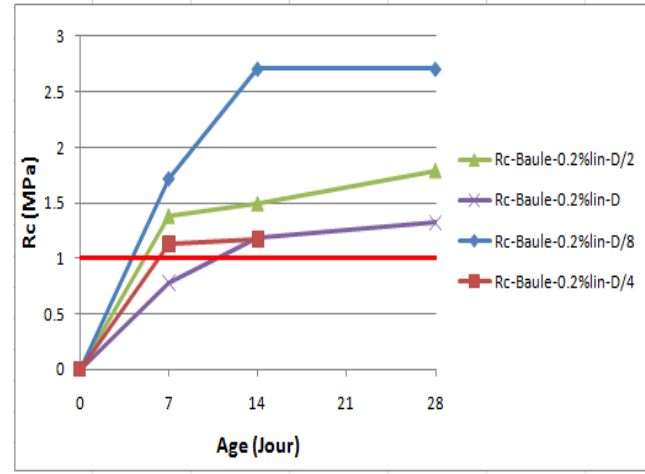

(b) Sédiments de La Baule - Le Pouliguen

Figure 5. Evolution de la résistance à la compression en fonction du temps.

Pour les sédiments de la Baule, la figure $5 \mathrm{~b}, \mathrm{Rc}$ croît de 7 jours à 14 jours, se stabilise et diminue aussi vers 28 jours. On constate aussi que tous les résultats de Rc dépassent le seuil de $1 \mathrm{MPa}$ mais restent en deçà des valeurs obtenues pour les fillers. Aussi toutes ces observations peuvent s'expliquer vraisemblablement par la forte teneur en matières organiques des sédiments de La Baule - Le Pouliguen $(\mathrm{MO}=11.5 \%)$ qui retarde ou peut carrément empêcher le processus d'hydratation du ciment.

\subsubsection{Influence du dosage et de la longueur des fibres}

Sur la figure 6, on note globalement que Rc est la plus élevée pour des longueurs D (34 $\mathrm{mm}$ ) et $\mathrm{D} / 2(17 \mathrm{~mm})$ et pour les dosages $0.8 \%$. D'autres dosages sont aussi à retenir étant donné la variation observée sur la figure 6. La nature des fibres jouent un rôle et ceci est aussi en relation avec l'adhérence.
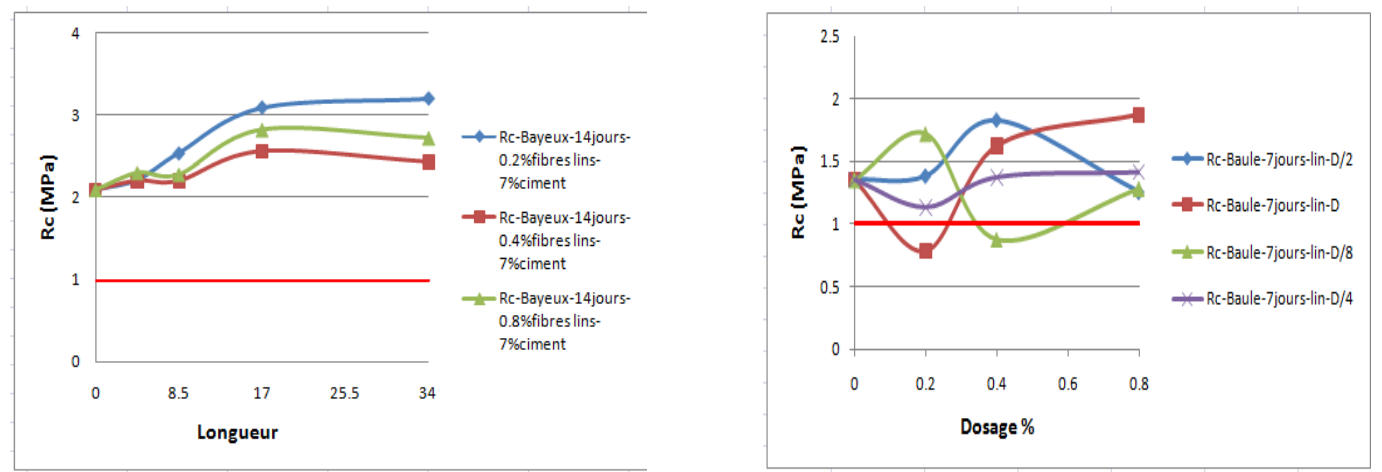

Figure 6. Effet du dosage et des longueurs de fibres sur Rc. 


\subsubsection{Effet de la nature des fibres}

En observant la figure 7, les résultats obtenus pour les fibres de lin sont les meilleurs pour les deux types de matériaux stabilisés, suivis par les fibres de kevlar (blanche) et ceux des fibres PP (noire). Ces observations peuvent s'expliquer par la répartition des fibres qui est plus homogène pour le lin que pour les fibres blanches ou noires, par la grande capacité d'imbrication du lin avec les gains des sols fins. Mais les fibres noires sont lisses, ce qui conduit à un frottement faible et au glissement des fibres entre les grains au moment de la mobilisation de la résistance encompression.
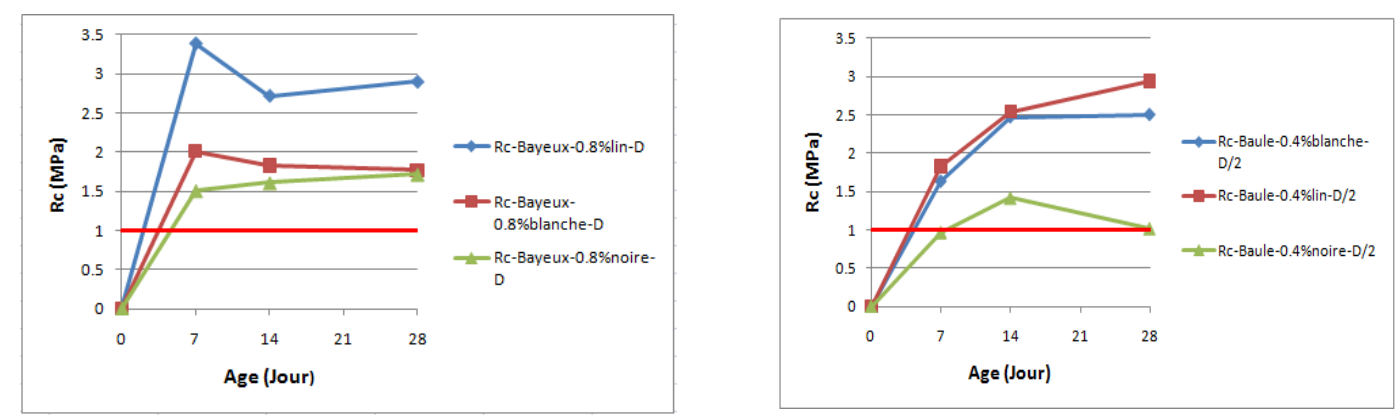

Figure 7. Effet de la nature des fibres sur la résistance à la compression.

\subsection{Etude des ruptures}

Si l'on s'intéresse au comportement en compression des échantillons, la figure 8 montre que, pour une éprouvette sans fibre, une rupture immédiate se produit de type fragile (fractures pseudo-parallèles) nommée rupture localisée. Puis pour les éprouvettes fibrées, les modes de rupture sont de type diffus. Pour ce type de rupture diffuse, on remarque que les fibres de lin à fort dosage conduisent un emboitement de différentes parties de l'éprouvette entre elles, indiquant un comportement très ductile. Ce type de rupture est appelée rupture diffusée emboitée (voir figure 8).

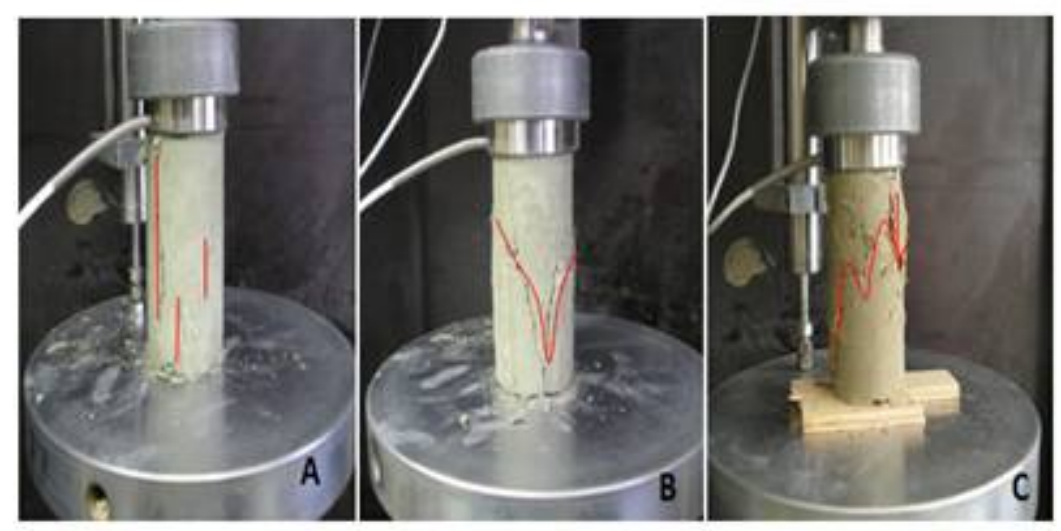

(A) Rupture localisée - (B) Rupture diffuse - (C) Rupture diffuse emboitée

Figure 8. Différents types de ruptures observés après un essai de compression simple. 


\section{XII ${ }^{\text {èmes }}$ Journées Nationales Génie Côtier - Génie Civil \\ Cherbourg, 12-14 juin 2012}

6.3 Etude de la performance mécanique vis-à-vis du domaine routier

La performance mécanique des formulations est pour un matériau routier, évaluée au travers du module d'élasticité et de la résistance en traction. Les résultats sont ensuite reportés sur l'abaque de classification de la norme NF P 981143.

Ceci confirme la possible utilisation en couche de forme de chaussée des mélanges proposés. La classification des différentes formulations étudiées pour les deux matériaux fins traités sur l'abaque, montre une qualification en S5, voir figure 9. Les matériaux classés en S5 ne peuvent pas prétendre à une utilisation en couches de formes selon (SETRA \& LCPC, 2000). Ce classement est dû à la finesse des grains des éléments traités et pour y remédier, il peut être proposé d'augmenter la granulométrie des matériaux.

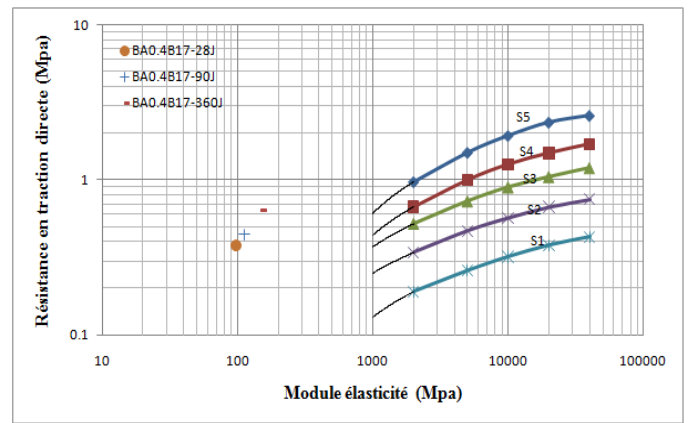

Figure 9. Classification de la formulation avec $0.4 \%$ de fibre kevlar (blanche), de longueur D/2 pour les sédiments de La Baule - Le Pouliguen, à 28, 90 et 360 jours.

\section{Conclusion}

Pour les mélanges des matériaux fins étudiés, on observe une amélioration des propriétés mécaniques à la rupture pour une quantité optimale de fibres de $0.8 \%$. On remarque que pour cette valeur, la résistance à la compression à la rupture est supérieure au seuil de 1 MPa requis dans le cas de stabilisation de matériaux fins en sous couche routière.

La quantité, la longueur et la nature des fibres utilisées dans les mélanges restent à optimiser. Le comportement des matériaux valorisés avec des fibres, est modifié. On remarque que les résistances la compression simple sont meilleures pour les fibres de lin devant les fibres en kevlar (blanche) et lisses en polypropylène.

\section{Références}

AIPCN (2006). La gestion des sédiments pollués en rivière. Source AIPCN, Disponible sir : http://www.cetmef.equipement.gouv.fr/aipcn/fichiers/sediments.doc

BASHA E.A., HASHIM R., MAHMUD H.B., MUNTOBAR A.S. (2005). Stabilization of residual soil with rice husk ash and cement. Construction and Building Materials, Vol. 19 (6), pp 448-453. doi:10.1016/j.conbuildmat.2004.08.001 
CHERIFI N. (2011). Covalorisation d'un sédiment marin et d'un filler dans une matrice ciment renforcée par des fibres. MSc report, Université de Caen, France, 73 p. GEETHAMMA V.G., MATHEW K.T., LAKSHMINARAYANAN R., SABU T. (1998). Composite of short coir fibres and natural rubber: effect of chemical modification, loading and orientation of fibre. Polymer, Vol. 39, Issues 6-7, pp 1483-1491.

GHAVAMI K., TOLEDO FILHO R.D., BARBOSA N.P. (1999). Behaviour of composite soil reinforced with natural fibres. Cement and Concrete Composites, Vol. 21 (1), pp 39-48. doi:10.1016/S0958-9465(98)00033-X

HEJAZI S.M., SHEIKHZADEH M., ABTAHI S. M., ZADHOUSH A. (2012). A simple review of soil reinforcement by using natural and synthetic fibers. Construction and Building Materials, Vol. 30, pp 100-116. doi:10.1016/j.conbuildmat.2011.11.045

KOTAKE N., URAYAMA S., MIYAWAKI F., KAMON M. (2012). Compression, tension and bending strength characteristics of fiber-reinforced cement treated soil, Geo-Environmental Engineering, GEE2012, Caen University, Caen, France, pp 77-84.

LE T.N.H. (2009). Comportement des sols fins utilisés en couverture étanche des installations de stockage de déchets. Approche expérimentale et numérique. $\mathrm{Ph} . \mathrm{D}$ Thesis, Université de Grenoble, France, 164 p.

LEVACHER D., WANG F., LIANG Y. (2010). Co-valorisation de matériaux fins équivalents à des sediments, JNGCGC 2010, Sables d'Olonne, pp 869-876, doi:10.5150/jngcgc.2010.094-L.

LEVACHER D., LIANG Y., CHERIFI N., BATAGIN B. (2012). Reuse of fibers in sediments and fillers stabilization: some experiments with cement-lime mixtures, GeoEnvironmental Engineering, GEE2012, Caen University, Caen, France, pp 179-186.

LIANG Y. (2012). Co-valorisation de sédiments et de sols fins par apport de liants et de fibres. Ph.D Thesis, Université de Caen, France, 166 p.

SERFASS J.-P., SAMANOS J. (1996). Fiber-modified asphalt concrete characteristics, applications and behavior. Journal of the Association of Asphalt Paving Technologies, Vol.65, pp 193-230. doi:10.1016/j.geotexmem.2006.11.002

SETRA, LCPC (2000). Guide des Terrassements Routiers, réalisation de remblais et des couches de formes, fascicules I et II, GTR 2ème édition Juillet 2000, 211 p.

TANG B., GAO W., CHEN F., CAI Y. (2007). Strength and mechanical behavior of short polypropylene fiber reinforced and cement stabilized clayey soil. Geotexiles and Geomembranes, Vol. 25, pp 194-202.

WANG F. (2008). Caractérisation de fines de carrière et de sédiments marins Amélioration de la résistance des fines de carrière par des procédés de solidification. MSc report, Université de Caen, France, 43 p. 